\title{
A cost-reducing extracorporeal membrane oxygenation (ECMO) program model: a single institution experience.
}

\author{
Nicholas C. Cavarocchi \\ Thomas Jefferson University \\ S Wallace \\ Thomas Jefferson University \\ E Y. Hong \\ Thomas Jefferson University \\ A Tropea \\ Thomas Jefferson University \\ J Byrne \\ Thomas Jefferson University \\ Follow this and additional works at: https://jdc.jefferson.edu/surgeryfp \\ Part of the Medical Sciences Commons

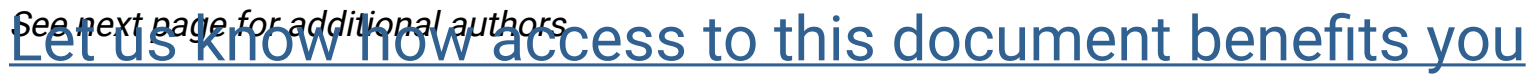

\section{Recommended Citation}

Cavarocchi, Nicholas C.; Wallace, S; Hong, E Y.; Tropea, A; Byrne, J; Pitcher, Harrsion; and Hirose, Hitoshi, "A cost-reducing extracorporeal membrane oxygenation (ECMO) program model: a single institution experience." (2015). Department of Surgery Faculty Papers. Paper 120. https://jdc.jefferson.edu/surgeryfp/120

This Article is brought to you for free and open access by the Jefferson Digital Commons. The Jefferson Digital Commons is a service of Thomas Jefferson University's Center for Teaching and Learning (CTL). The Commons is a showcase for Jefferson books and journals, peer-reviewed scholarly publications, unique historical collections from the University archives, and teaching tools. The Jefferson Digital Commons allows researchers and interested readers anywhere in the world to learn about and keep up to date with Jefferson scholarship. This article has been accepted for inclusion in Department of Surgery Faculty Papers by an authorized administrator of the Jefferson Digital Commons. For more information, please contact: JeffersonDigitalCommons@jefferson.edu. 
Authors

Nicholas C. Cavarocchi, S Wallace, E Y. Hong, A Tropea, J Byrne, Harrsion Pitcher, and Hitoshi Hirose 
A cost reducing extracorporeal membrane oxygenation (ECMO) program model: a single institution experience

En Yaw Hong, BS; Suzanne Wallace, CRNP; Amy Tropea, CCP; Jaime Byrne, CRNP; Harrison T. Pitcher, MD; Hitoshi Hirose, MD, PhD; Nicholas C. Cavarocchi, MD.

From Department of Surgery, Thomas Jefferson University, Philadelphia, Pa., USA.

Short running title: Interdisciplinary Intensive Care Run ECMO

This paper was presented at ASAIO's 59th Annual Conference. Chicago, IL. June 12-15, 2013.

Corresponding author: Nicholas C. Cavarocchi, MD.

Division of Surgical Cardiac Care Unit, Department of Surgery, Thomas Jefferson University 1025 Walnut Street Room 605, Philadelphia, PA 19107, USA.

Tel: 215-955-5654; Fax: 215-955-6010

E-mail: Nicholas.Cavarocchi@jefferson.edu

Word Count of main text: 2780

Key word: ECMO, ICU, cost, patient safety. 


\begin{abstract}
Background: The worldwide demand for ECMO support has grown. Its provision remains limited due to several factors (high cost, complicated technology, lack of expertise) that increase healthcare cost. Our goal was to assess if an intensive care unit (ICU) run ECMO model without continuous bedside perfusionists would decrease costs while maintaining patient safety and outcomes.
\end{abstract}

Method: New ECMO program implemented in 2010 consisted of a dedicated ICU involving multidisciplinary providers (ICU registered nurse, mid-level providers and intensivists). In year one, we introduced an education platform, new technology and dedicated space. In year two, continuous bedside monitoring by perfusionists were removed and new management algorithms designating multidisciplinary providers as first responders were established. The cost and patient safety of this new ECMO program was retrospectively collected and these were compared between year 1 and year 2 .

Results: During the study period, 74 patients (28 patients in year 1 and 46 patients in year 2) were placed on ECMO (mean days: $8+/-5.7$ ). The total annual hospital expenditure for the ECMO program was significantly lower in new model $(\$ 234,000$ in year 2 vs. $\$ 600,264$ in year 1), showing a $61 \%$ decrease in cost. This cost decrease was attributed to a decreased utilization of perfusionist services and the introduction of longer lasting and more efficient ECMO technology. We did not find any significant changes in registered nurse ratios or any differences in outcomes related to ICU safety events.

Conclusion: We demonstrated that the ICU-run ECMO model managed to lower hospital cost, by reducing the cost of continuous bedside perfusion support without a change in outcomes. 


\section{Introduction}

Extracorporeal membrane oxygenation (ECMO) is an advance life-support that incorporates the use of a cardiopulmonary bypass circuit to sustain the cardiovascular and pulmonary system via hemodynamic support and gas exchange. Given the historical complexities of its usage and dismal survival rates [1], an international consortium "Extracorporeal Life Support Organization (ELSO)" has been established to support ECMO centers through continuing education and guideline development. The guidelines consisted of the ideal institutional requirements for the effective use of ECMO including a proper organization structure, staffing issues, physical facilities and equipment, staff training and continuing education, selection criteria, patient follow-up and program evaluation [2]. Despite this, there is still a variation in the implementation of ECMO programs among centers, leading to the popular belief that the varying survival rates reported in literature are due to a nonstandardized approach especially in smaller institutions [3].

The failure of standardization among centers can be attributed to the lack of specialized ECMO trained staff (ECMO specialists). This shortage was highlighted during the H1N1 pandemic in 2009 when this patient population grew exponentially [4]. Since then, the demand for ECMO has steadily increased leading ELSO to develop new guidelines for ECMO centers in 2010. The new guidelines address the perfusionist shortage by allowing board certified nurses who have at least one year of critical care experience to train as ECMO trained staff specialists. Although this has been shown to expand ECMO availability, financial concerns have been reported in training all ICU providers' as potential ECMO specialists [5, 6]. The failure of the ELSO guidelines has been to not recognize that dedicated ECMO specialists at the bedside are 
not the answer; a dedicated ECMO educated team at the bedside with up to date technology addresses the shortage.

Despite this, the financial concerns were not weighed against the long-term cost benefit of training nurses as ECMO providers not specialists. Therefore, this study aims to describe the experiences of implementing a new cost-reducing ECMO program model in an ICU setting of an established adult ECMO center involving multidisciplinary providers (registered nurses, midlevel providers and intensivists) as ECMO providers. This study will also show the cost benefit of the new ECMO model in terms of hospital expenditure, patient safety and outcomes.

\section{Method}

The new ECMO model was introduced in July 2010 in our institution. An adult ECMO program was established with the consensus of the surgical cardiac critical care director, other intensivists, hospital and nursing administration, staff nurses, unit manager, clinical nurse specialist, and perfusionists. During the first year, new technology and an educational program were introduced for all levels of the staff.

New technology was introduced to replace and update the previously used components of the ECMO circuit. The Rota flow pump (Maquet Cardiovascular LLC, San Jose, California) was used to replace the Medtronic Biomedicus centrifugal pump. The Quadrox D, a lowpressure oxygenator (Maquet Cardiovascular LLC) was used to replace the Avecor oxygenators (Medtronic, Langhome, PA). The CardioHelp system (Maquet Cardiovascular LLC) was also introduced as a portable cardiopulmonary support system. Finally, Servo-I (Maquet Cardiovascular LLC) ventilator was introduced to transport and maintain difficult ventilation in ECMO patients. The circuit was simplified to include the oxygenator and Rota flow pump in a 
closed loop design. There were no points of access into the circuit, reservoir or pressure lines pre or post oxygenator. All cannula were inserted thru a percutaneous approach.

The education platform (Table 1) was developed by a multidisciplinary collaboration involving intensivists, perfusionists and nursing. An outside consultant who specialized in ECMO therapy in conjunction with the ICU nurse educator and perfusionists initially provided the education. The education was provided to registered nurses, mid-level providers, staff physicians, physical therapists and respiratory therapists. It consisted of three main components, which were didactic sessions, hands-on sessions in the wet-labs and competency tests. These requirements were summarized in a competency checklist that the trainee had to complete by the end of the training course. The didactic sessions mainly focused on the existing fundamental knowledge on ECMO and cardiopulmonary physiology. Included in these didactic sessions were discussions related to the institutional adult ECMO policy and procedures including cardiac and pulmonary indications, common complications, ECMO contraindications, patient and circuit monitoring, ECMO troubleshooting procedures and emergency management protocols. Simulation training or hands-on sessions were held in a dedicated space in the hospital allocated for the training of all ECMO providers and was termed wet-labs (saline primed ECMO circuit). During these sessions, the trainees learned to operate the ECMO console and tubing system including hand cranking for emergency situations. Both the didactic sessions and wet-labs were taught by a chief perfusionist, an outside consultant and the clinical nurse educator. At the completion of the training process, trainees were required to undergo a competency test. This test and training was held annually even after the completion of the initial training course to ensure continuous competency among the ICU staff. Each trainee's competency checklist was also assessed for completion. The goal of the education platform was to provide knowledge and 
experience in monitoring the ECMO circuit and patient, troubleshooting procedures and identifying and preventing complications.

The second major component of the ICU-run ECMO model included the allocation of a dedicated space; the development of treatment algorithms for ECMO providers, the introduction of ECMO trained personnel and the reduction of bedside responsibility by the perfusionists'. The allocation of a dedicated space for the ECMO patients' was decided upon by room availability, accessibility and location size factors. Based on these factors, a dedicated cardiovascular ICU was selected as the best location for the implementation of the model.

The algorithms developed included protocols for anticoagulation, hemodynamic management, oximetry, nutrition, ventilation management, surgical interventions, ECMO weaning criteria, and requirements for transport of patients (both within the hospital and outside of the hospital) who require ECMO support. These algorithms were developed by the unit director and modified accordingly based on feedback given by ECMO providers, nurses, residents and others. Concerns and suggestions for the algorithms were addressed in multidisciplinary conferences involving the unit director, intensivists, residents, perfusionists and, mid-level providers, and nurses. These were held regularly during the initial implementation of the program to ensure quick and effective evaluation and modification of the treatment algorithms. The unit director also conducted daily rounds to ensure proper implementation of the treatment protocols.

The second year, began with the weaning of continuous bedside monitoring by the perfusionists from the bedside. This marked the transition of responsibilities as it related to the ECMO patient. The perfusionists remained responsible for priming the circuit and support during the initial start up of therapy. Initially, the ECMO patients had a 2:1 nurse to patient ratio (same 
for both years) for the first 12-24 hours. After the patient was stable on ECMO support, the perfusionist was not required to stay at the bedside or in-hospital. The ICU nurse became responsible for the patient and monitoring the effectiveness of ECMO therapy and for any problems with the circuit. After the transition of care, the perfusionists were required to be to be available to troubleshoot any circuit related issue. Since many patients were supported on ECMO for longer than a day (mean duration of support was 8 days), the perfusionists were required to conduct daily rounds on the patients and assist in management strategies, troubleshooting and assessment. The bedside nurse was responsible for ongoing management and identification of patient or circuit related problems. If the patient required an intervention to either clinical care or circuit, the unit based mid-level provider was notified. The mid-level provider was able to prescribe changes to the ECMO therapy within their scope of practice, this included speed, sweep or FiO2 adjustments. If there was a problem with the circuit that required manipulation, adjustment or exchange of a component, the perfusionist and Intensivist on call would be notified and respond appropriately. This ECMO model achieved completion in July 2012.

To measure the cost benefit of the new ICU run ECMO model, adult patients who required ECMO therapy in our institution from July 2010 to July 2012 were retrospectively reviewed after approval by institutional review board.-Pediatric patients (17 years old and below) and those who were transferred from outlying hospital centers already on ECMO support were excluded from this study. Following variables were collected: demographic factors (age, gender, body mass index), primary diagnosis necessitating ECMO, associated medical history, SOFA score and APACHE II score, and duration of treatment. The patients were divided into 2 groups; the service model of ECMO during the first year (ECMO with the perfusionist with new 
technology and education - Group 1); the new ECMO model during the second year (ICU managed with new technology and education, Group 2).

The hospital cost incurred by ECMO patients (cost of ECMO set-up and cost of continuous perfusion monitoring between service models) was compared between 2 groups. Hospital accounting and billing records were reviewed to obtain the cost to set up an ECMO circuit and perfusionists' monitoring fee. The perfusion services at this institution involve an outside vendor who provides services on a contract basis. The hospital cost to set up an ECMO circuit was $\$ 834$ and the perfusionists' monitoring fee was $\$ 101 /$ hour. A monthly retainer fee introduced in the $2^{\text {nd }}$ year replaced the hourly fee schedule. This fee included the training and development of nurse competencies and setting up of ECMO circuit. The cost to set up ECMO and the perfusionists' hourly monitoring fee were only applicable for the first year, while the monthly retainer fee was only applicable for the second year. The mean annual costs during the $1^{\text {st }}$ and $2^{\text {nd }}$ year were calculated and compared. The number of safety issues (complications derived from incompetency) and outcomes (mortality) were compared between 2 groups.

Data were expressed with number, percentage or mean with standard deviation as appropriate. Statistical analyses were performed Stata (Stata Co, College Station, TX). Twogroup comparison was performed with Chi Square or Student's t-tests, Mann-Whitney U test as appropriate. $\mathrm{P}$ value less than 0.05 were considered significant.

\section{Result}

During the study period, a total of 74 patients were placed in ECMO in our institution and all were included in the study.- The basic patient characteristics are shown in Table 2.

Of the 74 patients, $38 \%(n=28)$ were treated during the 1 st year of the ECMO model (group 1) while the remaining 62\% (n=46) were treated during the 2 nd year (group 2). There 
were no significant differences in patient's demographics, medical history, primary diagnosis, type of the ECMO device, and duration of the ECMO therapy between the two groups. The patients in-group 2 were sicker compared to group 1 (higher APACHE II and SOFA score); however there was no significant mortality differences between 2 groups.

In-group 1, the annual total cost of the ECMO set up was $\$ 23,352$; the annual total perfusionists' monitoring fee was $\$ 576,912$. In year 1 the total annual ECMO cost incurred from group 1 was $\$ 600,264$. In year 2 , the total retainer fee for group 2 was $\$ 234,000$. This fee includes the ECMO circuitry with no additional monitoring fee; the total annual ECMO cost incurred from group 2 was only $\$ 234,000$. Therefore, group 2 led to an annual cost savings of $\$ 366,264$ (Table 3).

The mortality rates between 2 groups were not significantly different $42 \%(n=12)$ ingroup 1 versus $59 \%(\mathrm{n}=27)$ in-group 2, $\mathrm{p}=0.233)$. Circuit exchange due to thrombus in the oxygenator was observed $11 \%(n=3)$ in-group 1 and $11 \%(n=5)$ in-group $2(p=0.650)$. In terms of safety events, an isolated event of dislodged central cannula was observed in-group 2. Upon review, it was deemed that the event did not occur because of incompetence of ICU staff but it was related to the loosely inserted cannula into the atrium. No other adverse safety event occurred in either group.

\section{Discussion}

Ever since the inception of non-perfusionist personnel in the monitoring of ECMO patients, there were mixed opinions in the benefits of implementing a non-perfusionist run ECMO model $[5,6,7]$. The University of Michigan reported an expansion in the availability of ECMO with no adverse safety events or complications related to nurses in the Primary Care Group (PCG) model [5]. In addition, they were able to allocate more staffing members to 
manage ECMO in lung transplant patients. On the other hand, concerns on the additional time needed to train new ECMO specialists and the possibility of adverse events deterred an institution from recruiting non-perfusionist staff as ECMO specialists [7]. The nurses in a Scottish ICU who were new ECMO specialists also reported the feeling of inadequacy and the need for regular competency tests to maintain their skills in managing the ECMO [6].

Nevertheless, there was a general consensus that implementing a non-perfusionist run ECMO model would require additional financial resources.

In our study, we found that there was significant cost savings in training ICU staff to care for and manage patients supported on ECMO, without an additional person dedicated to monitoring the ECMO circuit. This was mostly due to the omission of the perfusionists' monitoring fee. Although our institution was required to pay a fix fee per month (monthly retainer fee) regardless of the presence of ECMO cases, our total end cost was still cheaper compared to the total cost of the previous model. This was because of the increase in ECMO circuit availability and therefore increases in the number of ECMO patients in the new model. It was calculated that the minimum duration of ECMO for this model to be cost beneficial was 7.7 days (our mean ECMO duration was 7.9 days) assuming a rate of one patient per month.

Although we are a registered ECMO center in the country, our institution has a moderate number of ECMO patients compared to other larger ECMO institutions. This could potentially lead to the lack of confidence and the erosions of skills in handling ECMO patients [6]. To prevent this, we conducted regular competency tests and training courses. These competency tests and training courses have helped the multidisciplinary group of ECMO providers to maintain their skills and gain confidence in handling ECMO patients. In addition, the open 
relationship between the perfusionists and ICU staff helped all feel secure, as there was someone they can turn to if a complication arises.

From the perfusionists' perspective, the introduction of this new ECMO model has allowed them to allocate more time in the operating room. Initially, they were hesitant to transition continuous care to the ICU model, and they were not confident in the ICU teams capability in handling ECMO patients. However, after observing the success of the new team approach, they felt that the model has streamlined and simplified their duties.

There are those that are still concerned about this model for various reasons. First, that anyone other than a perfusionist "managing an ECMO circuit" may be outside the scope of practice. Second, that a patient is unsafe with "nurses managing the ECMO circuit". The keys to achieving the clinical outcomes presented above should not be overlooked. Nurses were not asked to manage the circuit, but rather, were asked to manage the patient and monitor the circuit similar to that of a ventricular assist device. Similarly, the perfusionists were still solely responsible for the management of the system. They exchanged the circuit or oxygenator if required, they went on transports outside the unit and they were present for both initiation and weaning of support. The circuit was set up to be simple and minimize the risk of circuit related complications. Without the change to updated technology, the ICU model for management of patients on ECMO should not be considered.

There were several limitations to this study. Firstly, the number of patients involved in this study was limited. A larger sample size could potentially increase the amount of cost savings, as the monthly retainer fee is constant. . Secondly, the diversity of disease processes managed did not allow for comparisons between respiratory vs. cardiac failure. Finally, we employed a third party to train ICU staff and perfusionists. This could potentially lead to 
additional financial biases. We ensured that this was not the case as they were not involved in any decision making in this model and were never present in any of the meetings and discussions that took place during the implementation of the new ECMO model.

\section{Conclusion}

The new ICU run ECMO program implemented in our study is cost beneficial with no adverse safety events or complications related to new ICU managed ECMO model. The introduction of this model has managed to expand availability of ECMO, which influenced costsavings. The new ICU run ECMO program is truly exciting because the known high cost of managing an ECMO patient could be reduced without implications to outcomes. 
Table 1: New ECMO specialists' competency checklist

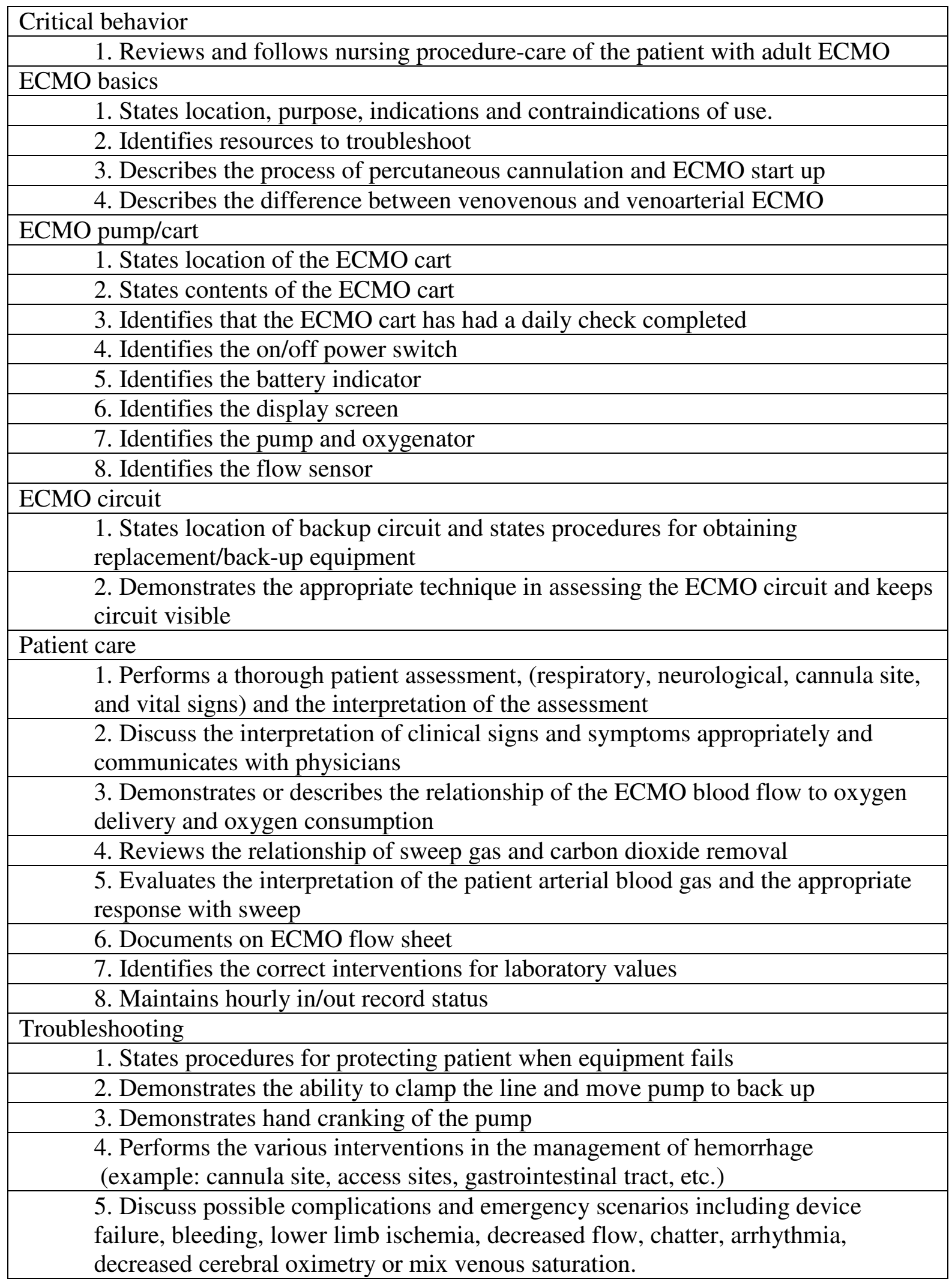


Table 2: Patients' baseline characteristics. Data are expressed with mean \pm standard deviation or number (percentage).

\begin{tabular}{|l|c|c|l|}
\hline & Group 1 (n=28) & Group 2 (n=46) & P-value \\
\hline Age (years) & $44 \pm 14$ & $49 \pm 14$ & 0.183 \\
\hline Males & $14(50 \%)$ & $24(52) \%$ & 0.636 \\
\hline Body mass index & $27.9 \pm 7.7$ & $31.5 \pm 8.6$ & 0.077 \\
\hline Tobacco & $6(21 \%)$ & $18(39 \%)$ & 0.133 \\
\hline Coronary artery disease & $13(46 \%)$ & $15(33 \%)$ & 0.323 \\
\hline Diabetes & $12(43 \%)$ & $16(35 \%)$ & 0.610 \\
\hline Chronic lung disease & $3(11 \%)$ & $3(7 \%)$ & 0.622 \\
\hline Cardiogenic shock & $20(71 \%)$ & $34(74 \%)$ & 0.510 \\
\hline Respiratory failure & $7(25 \%)$ & $9(20 \%)$ & 0.577 \\
\hline SOFA & $12 \pm 2.2$ & $13 \pm 2.6$ & 0.051 \\
\hline APACHE II & $29 \pm 4.8$ & $23 \pm 6.9$ & 0.004 \\
\hline Venoarterial ECMO & $23(82 \%)$ & $39(85 \%)$ & 0.757 \\
\hline Duration of ECMO days & $8.5 \pm 5.7$ & $7.9 \pm 6.9$ & 0.695 \\
\hline Mortality & $12(42 \%)$ & $27(59 \%)$ & 0.233 \\
\hline
\end{tabular}


Table 3: Details of ECMO cost of group 1 vs. group 2

\begin{tabular}{|l|l|l|l|}
\hline & Cost & $\begin{array}{l}\text { Incidence per } \\
\text { year }\end{array}$ & Total \\
\hline Fee per ECMO set up & $\$ 834$ & 28 & $\$ 23,352$ \\
\hline Fee per hour & $\$ 101$ & 5712 & $\$ 576,912$ \\
\hline Total ECMO cost by group 1 & & & $\$ 600,264$ \\
\hline & & & \\
\hline & & $\begin{array}{l}\text { Incidence per } \\
\text { year }\end{array}$ & Total \\
\hline Group 2 & Cost & 12 & $\$ 234,000$ \\
\hline Fee per monthly retainer & $\$ 19,500$ & & $\$ 234,000$ \\
\hline Total ECMO cost by group 2 & & & \\
\hline & & & $\$ 366,264$ \\
\hline ECMO savings & & & \\
\hline
\end{tabular}




\section{Reference}

1. ELSO registry report international summary, January 2013. Available from Extracorporeal Life Support Organization web site: http://www.elsonet.org/ (Accessed 1 January 2014).

2. ELSO guidelines for ECMO centers. Available from Extracorporeal Life Support Organiza tion web site. http://www.elsonet.org/ (Accessed 1 January 2014).

3. Crow S, Fischer AC, Schears RM. Extracorporeal life support: utilization, cost, controversy, and ethics of trying to save lives. Semin Cardiothorac Vasc Anesth 2009; 13: 183-191.

4. H1N1 information. Available from Extracorporeal Life Support Organization web site. htt p://www.elso.med.umich.edu/H1N1Info.html (Accessed 1 January 2014).

5. Freeman R, Nault C, Mowry J, Baldridge P. Expanded resources through utilization of a primary care giver extracorporeal membrane oxygenation model. Crit Care Nurs Q2012; 35: 39-49.

6. Berryman S. Extracorporeal membrane oxygenation in a Scottish intensive care unit. Nurs Crit Care 2010; 15: 262-268.

7. Mongero L, Beck J, Charette K. Managing the extracorporeal membrane oxygenation (ECMO) circuit integrity and safety utilizing the perfusionist as the "ECMO Specialist" Perfusion 2013; 28: 552-554. 